\title{
One lung, two coronary stents and three centimeters malignant brain tumour! tailored anaesthesia for a lethal combination
}

\begin{abstract}
When patient-related concerns are multiple, the anesthetic considerations also multiply. Stereotactic neurosurgery is done for diagnostic and/or therapeutic purposes for selective brain lesions and may require prolonged general anesthesia. It involves three-dimensional localization of lesions (for biopsy/treatment) utilizing a specialized stereotactic localization head frame system. Airway management (both mask ventilation and endotracheal intubation) becomes difficult with the frame in situ. We hereby present a known hypertensive patient with raised intracranial tension for stereotactic neurosurgery. Our patient also had two coronary artery stents, following previous myocardial ischemia. A major anesthetic challenge was posed by the presence only one lung in this patient, following a leftsided pneumonectomy done for bronchogenic carcinoma. This case report highlights the successful anesthetic management of such a complex case with judicious ventilatory and hemodynamic optimization.
\end{abstract}

Keywords: stereotactic neurosurgery, post pneumonectomy, post percutaneous coronary angioplasty, malignant space occupying lesion, raised intracranial pressure
Volume 6 Issue 4 - 2016

\author{
Shah SB,' Dubey $M,{ }^{2}$ Bhargava AK, ${ }^{3}$ Uma \\ Hariharan4 \\ 'Consultant Anaesthesiologist, Rajiv Gandhi Cancer Institute and \\ Research Centre, India \\ ${ }^{2}$ Senior Consultant, Rajiv Gandhi Cancer Institute and Research \\ Centre, India \\ ${ }^{3}$ Director Anesthesiology, Rajiv Gandhi Cancer Institute and \\ Research Centre, India \\ ${ }^{4}$ Fellowship Oncoanaesthesia, Assistant Professor Anaesthesia \\ and Intensive Care, Dr. Ram Manohar Lohia Hospital and \\ PGIMER, India
}

\begin{abstract}
Correspondence: Shagun Bhatia Shah, Consultant Anaesthesiologist, Rajiv Gandhi Cancer Institute and Research Centre, H. No: 174- 175, Ground Floor, Pocket - 17, Sector-24, Rohini, Delhi- I 10 085, India, Tel 9891769779; 987। 347836, Email drshaganר_2010@rediffmail.com
\end{abstract}

Received: May 15, 2016 | Published: December 16, 2016

\section{Introduction}

Stereotactic neurosurgery/craniotomy for malignant tumour decompression ${ }^{1}$ in a cardiac patient with single lung poses myriad challenges. Anesthetic management has to be tailored for optimizing individual organ function. Maintenance of three important pressures ${ }^{2}$ namely intracranial pressure, blood pressure and the airway pressure are vital in such a scenario. Stereotactic neurosurgery was done in this patient for brain metastasis, which developed despite trimodal ${ }^{3}$ therapy (surgery, chemotherapy and radiotherapy) for bronchogenic carcinoma. ${ }^{4}$ A practical, protocol-based anaesthetic technique was used for tumour decompression neurosurgery lasting six hours in this challenging patient. Optic nerve sheath diameter was utilized for noninvasive measurement of intracranial pressure. ${ }^{5}$ Anesthetic considerations $^{4}$ for combined post-pneumonectomy and postcoronary artery stenting situation are hereby highlighted in this unique case report.

\section{Case report}

A 61 years old, $63 \mathrm{~kg}$, height $174 \mathrm{~cm}$, male patient, diagnosed with left bronchus carcinoma with brain metastasis who presented with headache and progressive left hemiparesissince last 14 days. He had undergone left pneumonectomy (Figure 1) followed by 6 cycles of chemotherapy (gemcitabine, cisplatin) and 10 fractions of radiotherapy (39 grey total) 18 months ago. The patient had undergone percutaneous trans luminal coronary angioplasty (with placement of 2 bare metal stents) 5 years ago. Echocardiography revealed mild mitral valve prolapse, mitral regurgitation, tricuspid regurgitation and moderate pulmonary artery hypertension (right ventricular systolic pressure: $46 \mathrm{mmHg}$ ). Dobutamine stress echocardiography was negative for provocable ischemia at $81 \%$ of heart rate. Aspirin had been discontinued a week earlier. His haematological and biochemical investigation reports were within normal limits except for mild hyponatremia (serum sodium $133 \mathrm{mEq} / \mathrm{l}$ ). Whole body PET scan was suggestive of metabolically active right deep parietal periventricular brain lesion with peri lesional oedema and mass effect (Figure 1).

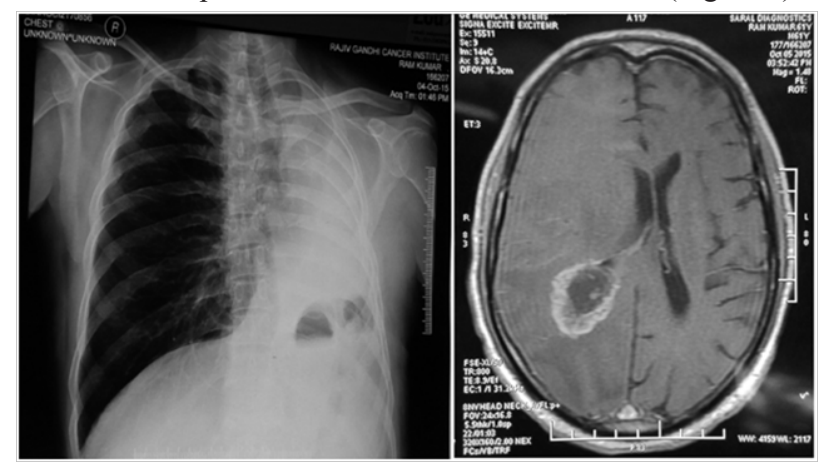

Figure I Chest Xray showing post left pneumonectomy changes and CTscan of brain with metastatic tumour causing midline shift.

Hemiparesis prevented the patient from performing pulmonary function tests satisfactorily. His preoperative arterial blood gases (ABG) in room air were within normal limits and the pulse oximeter displayed an SPO2 of $98 \%$ on room air. Preoperative optimization of the patient was done with intravenous mannitol $100 \mathrm{ml}$ every 8hours and dexamethasone $8 \mathrm{mg}$ every 8 hours, oral acetazolamide $250 \mathrm{mg}$ and levetiracetam $500 \mathrm{mg}$ twice a day, and metoprolol $25 \mathrm{mg}$ once a day were used. In the operation theatre, the right radial artery was cannulated and a cardiac output monitor (FloTrac/VigileoTM; Edwards Lifesciences, Irvine, CA, USA) was attached. A central venouspressure line was secured and all routine monitors along with peripheral nerve stimulator and entropy were attached. Invasive blood pressure guided dobutamine infusion was started at the priming 
dose for aiding myocardial contractility and preventing hypotension. Preoxygenation was done after turning the elbow connector $180^{\circ}$, so that the circuit tubing did not impact the stereotactic localization frame (Figure 2). Anaesthesia was induced with intravenous thiopentone $300 \mathrm{mg}$, fentanyl $100 \mu \mathrm{g}$ and atracurium $50 \mathrm{mg}$. The trachea was intubated with a $7.5 \mathrm{G}$ flexometallic tube using a CMac D Blade videolaryngoscope, followed bycapnography confirmation of ETT placement, nasogastric tube and oral pack insertion. The optic nerve sheath diameter (ONSD) was sonographically calculated as $6.1 \mathrm{~mm}$ in right eye and $5.9 \mathrm{~mm}$ in left eye. The patient was now placed in the left lateral position and the head fixed within the Mayfield adult horseshoe headrest system and skull clamp cradling and stabilizing the head off the end of the operating table and allowing the surgeon better access during surgery (Figure 2). Intravenous dexamethasone $8 \mathrm{mg}$ was injected. Infusions of mannitol $1 \mathrm{gm}$, levetiracetam $(500 \mathrm{mg}$ over 15minutes), entropy guided propofol and peripheral nerve stimulator directed atracurium were started. Anaesthesia was maintained with isoflurane $(\mathrm{MAC}<1)$ in medical air and oxygen. Goal directed fluid therapy was followed, keeping the central venous pressure (CVP) $8-10 \mathrm{mmHg}$ and stroke volume variation (SVV) less than 10. A total of $800 \mathrm{ml}$ of crystalloids (Plasma-Lyte ATM; Baxter Healthcare, Deerfield, Illinois.) were infused. The patient was mechanically ventilated using pressure controlled mode. Peak airway pressure was maintained at $22 \mathrm{mmHg}$ and the $\mathrm{ABG}$ (arterial blood gas analysis) showed a PaO2/FiO2 ratio of 703 and lactate of $1.7 \mathrm{mmol} / 1$. After the surgery and removal of head frame, muscle relaxant was reversed with intravenous neostigmine $2.5 \mathrm{mg}$ and glycopyrrolate $0.4 \mathrm{mg}$. Emergence was smooth and complete, with a postoperative heart rate of 56beats/min and blood pressure $129 / 86 \mathrm{~mm} \mathrm{Hg}$, The total procedure lasted 6 hours. An ONSD of 4.9 in both the eyes was recorded 4 hours postoperatively, suggestive of a normal intracranial pressure.

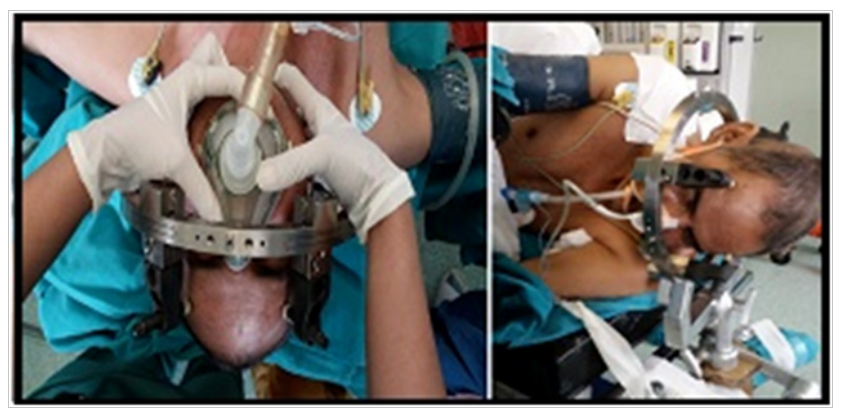

Figure 2 a) Two handed face masking (CE technique); b) Left lateral positioning of the patient with stereotactic frame in situ.

\section{Discussion}

A stereotactic localization frame $e^{1}$ in situ physically creates difficulty in mask ventilation and laryngoscopy, hampers neck mobility and may transform an anatomically normal airway into a difficult airway. This was overcome by rotating the elbow connecter, two hand C-E face masking technique and using a C Mac D blade videolaryngoscope ${ }^{2}$ (does not require alignment of the oral, pharyngeal and laryngeal axes) for inserting the flexometallic tube. The $\mathrm{C}$ Mac videolaryngoscope is extremely beneficial in such difficult airway circumstances. ${ }^{6}$ The D-blade also reduced the hemodynamic changes and was beneficial considering the cardiac status of the patient

Post pneumonectomy status ${ }^{4,5,7,8}$ results in a decrease in lung compliance and an increase in airway resistance. Mild to moderate exercise or a hyperdynamic condition(sepsis) coupled with the reduced capillary surface area, diminishes the capacity of the pulmonary capillaries to accommodate higher flow without raising pulmonary arterial pressure ${ }^{3}$ (hence the moderate increase in right ventricular systolic pressure in our patient with a single lung). We avoided nitrous oxide as it is known to aggravate pulmonary hypertension. Increased blood flow through the reduced pulmonary capillary bed and resultant increased pulmonary pressure may lead to pulmonary edema which is a serious concern in the post pneumonectomy patient and may influence mortality. Although pulmonary edema may worsen lung function, it reduces the pressure in the pulmonary capillary bed by the transudation of fluid into pulmonary interstitium. This effectively reduces the circulatory volume and offloads pressure generated in the right ventricle which if not relieved leads to right heart failure and mortality. ${ }^{3}$ Hence fluid restriction (we infused only $800 \mathrm{ml}$ of a physiological non lactated crystalloid is the key to avoiding pulmonary edema by limiting blood flow through the reduced pulmonary capillary bed. Fluid overload may be avoided with goal directed fluid therapy and diuretics as in our case. Care should be taken to avoid inadvertent endobronchial intubation on the same side of the pneumonectomy. The mechanical ventilator mode and settings are critical because lung edema will ultimately decrease lung compliance and cause Atelectasis. ${ }^{3}$ Setting a lower airway pressure (pressure controlled ventilation mode) is important to minimize pressure on the bronchial stump. At the same time, a nonjudicious reduction in airway pressure may also result in Atelectasis. ${ }^{9}$ If the remaining lung becomes atelectatic, the falling arterial blood saturation may force the anesthetist to increase airway pressure for gas exchange and alveolar recruitment, increasing the risk of bronchopleural fistula or rupture of the bronchial stump. ${ }^{9}$ Ventilatory modes that raise mean airway pressure for alveolar recruitment such as high frequency oscillatory ventilation (HFOV) or airway pressure release ventilation (APRV) may be contemplated. ${ }^{10}$ Systemic hypertension in a post-pneumonectomy patient can adversely affect peripheral oxygen delivery, producing heart strain, and precipitate pulmonary edema requiring prompt treatment. After load reducing agents, such as sodium nitroprusside (balanced arterial and venous dilatation) or hydralazine (arteriolodilator which increases cardiac output), may be used to treat systemic hypertension. ${ }^{11}$

Dobutamine infusion was started to improve the energetics of the right ventricle. Dobutamine and other beta-agonists are known to benefit patients exhibiting systemic hypoperfusion and also improve lung edema clearance, ultimately improving lung compliance. Levosimendan, a newer alternative, exhibits similar inotropic effects as dobutamine, additionally producing direct pulmonary vasodilatation. ${ }^{12}$ Systemic oxygen demands are hence met improving cardiopulmonary function. Serial ABGs and chest X-rays are useful in monitoring oxygenation, ventilation, and alveolar recruitment. Bronchoscopic removal of secretions or visualization of the bronchial stump maybe required.

Osmotic diuretic mannitol, dexamethasone and keeping inhalational anesthetic (isoflurane) at less than 1MAC (Minimum Alveolar Concentration) are the conventional measures of controlling raised intracranial pressure. Levetiracetam was used for seizure prophylaxis as it selectively prevents hyper-synchronization of epileptiform burst firing and propagation of seizure activity. Its oral and injectable formulations have equal bioavailability. Reduction in ONSD $^{5}$ recorded 4hours postoperatively was an indication that the surgery and anesthetic management of raised intracranial pressure was optimal. Optic nerve sheath diameter is being monitored in the recent times as an accurate non-invasive measure of the intracranial pressure, which can be extremely useful in both neurosurgical and non-neurosurgical procedures. 


\section{Conclusion}

Rotating the elbow connecter and using a C Mac D Blade videolaryngoscope are good measures for anticipated difficult airway due to presence of stereotactic localization mask in situ. Goal directed fluid therapy, diuretics and ionodilators (drugs with positive inotropic effect on the myocardium that simultaneously dilate the peripheral blood vessels) like dobutamine and levosimendan are useful in preventing right heart strain and pulmonary edema in post pneumonectomy patients. Airway pressure must be optimized to prevent breakdown of bronchial stump, at the same time preventing alveolar atelectasis (monitored by $\mathrm{PaO} 2 / \mathrm{FiO} 2$ ratio and serum lactate levels in ABG). Afterload reducing agents, such as nitroprusside or hydralazine, may be used to treat systemic hypertension. ONSD for noninvasive ICP monitoring is useful to guide anesthetic management. An understanding of the physiological consequences of both pneumonectomy and raised intracranial pressure allowed us to implement measures to preserve the function of the single remaining lung during this prolonged stereotactic neurosurgery in a cardiac patient.

\section{Acknowledgments}

None.

\section{Conflicts of interest}

The authors declare there are no conflicts of interest.

\section{Funding}

None.

\section{References}

1. Gibon D, Coste E, Vial S, et al. Stereotactic Localization in Medical Imaging: A Technical and Methodological Review. Journal of Radiosurgery. 1999;2(3):167-171.
2. Cavus E, Neumann T, Doerges V, et al. First clinical evaluation of the C-MAC D-Blade videolaryngoscope during routine and difficult intubation. Anesth Analg. 2011;112(2):382-385.

3. Kopec S, Irwin R,Umali-Torres $\mathrm{C}$. The postpneumonectomy state. Chest. 1998;114(4):1158-1184.

4. Veen EJ, Janssen-Heijnen MLG, Ritchie ED, et al. Pneumonectomy for bronchogenic carcinoma: analysis of factors predicting short- and long-term outcome. Interactive Cardiovascular and Thoracic Surgery. 2009;9:260-264.

5. Shah SB, Bhargava AK, Choudhury I. Noninvasive intracranial pressure monitoring via optic nerve sheath diameter for robotic surgery in steep Trendelenburg position. Saudi J Anaesth. 2015;9(3):239-246.

6. Shah SB, Hariharan U, Bhargava AK. C Mac D Blade: Clinical trips and Tricks. TACC. 2016;6:6-10.

7. KawaguchiT, Tojo T, Kushibe K, et al. Short- and long-term outcomes after pneumonectomy for primary lung cancer. Ann Thorac Cardiovasc Surg. 2008;14(5):289-293.

8. Massard G, Lyons G, Wihlm JM, et al. Early and long-term results after completion pneumonectomy. The Annals of Thoracic Surgery. 1995;59(1):196-200.

9. Tsuchida S, Engelberts D, Peltekova V, et al. Atelectasis causes alveolar injury in nonatelectatic lung regions. Am J Respir Crit Care Med. 2006;174(3):279-289.

10. Brambrink A, Brachlow J, Weiler N, et al. Successful treatment of a patient with ARDS after pneumonectomy using high-frequency oscillatory ventilation. Intensive Care Med. 1999;25(10):1173-1176.

11. Mason DT. Afterload reduction and cardiac performance. Physiologic basis of systemic vasodilators as a new approach in treatment of congestive heart failure. Am J Med. 1978;65(1):106-125.

12. Hill NS, Roberts KR, Preston LR. Postoperative pulmonary hypertension: Etiology and treatment of a dangerous complication. Respir Care. 2009;54(7):958-968. 